Supporting Information for:

\title{
Rapid Identification of Synthetic Routes to Functional Metastable Phases Using X- Ray Probed Laser Anneal Mapping (XPLAM) Time-Temperature Quench Maps
}

Authors: Robert T. Bell $1^{1,2 \dagger^{*}}$, Peter A. Beaucage ${ }^{1,3,4 \dagger^{*}}$, Marc J. Murphy ${ }^{1 \dagger}$, Aine B. Connolly ${ }^{1}$, Ulrich Wiesner ${ }^{1}$, David Ginley ${ }^{2}$, R. Bruce van Dover ${ }^{1}$, Michael O. Thompson ${ }^{1}$

\section{Affiliations:}

${ }^{1}$ Department of Materials Science and Engineering, Cornell University, Ithaca, NY 14853.

${ }^{2}$ Materials Science Division, National Renewable Energy Laboratory, Golden, CO 80401.

${ }^{3}$ Materials Science \& Engineering Division, ${ }^{4}$ NIST Center for Neutron Research, National Institute of Standards and Technology, Gaithersburg, MD 20877.

*Correspondence to: RTB (robert.bell@nrel.gov), PAB (peter.beaucage@nist.gov)

†These authors contributed equally to this work 


\section{Additional Methods:}

\section{Deposition}

Sample films of $\mathrm{Bi}_{2} \mathrm{O}_{3}$ were prepared as described in the Materials and Laser Annealing section of the main text. To verify that the bismuth oxide did not undergo stoichiometric shifts during laser processing, X-ray photoelectron spectroscopy was used to probe both the Bi oxidation state (Fig. S6) as well as the overall $\mathrm{Bi} / \mathrm{O}$ ratio in the films; the measurements gave atomic fractions of $\approx 65 \%$ oxygen for a furnace-annealed sample as compared with $\approx 62 \%$ for an area-annealed sample, well within the uncertainty of the measurement and consistent with a stoichiometry of $\mathrm{Bi}_{2} \mathrm{O}_{3}$.

\section{X-Ray Diffraction}

Diffracted X-rays were collected on a Pilatus3 300K pixel array detector (Dectris, Inc.) and radial integration was performed using the Nika package for Igor Pro (28). The peak markings in Fig. 2c correspond to International Crystal Structure Database (FIZ Karlsruhe) entries 2374, 41764, and 98144 for $\alpha, \beta$, and $\delta$, respectively. The beta and delta patterns were modified with an isotropic strain to better match the experimental data $(\approx-2 \%$ for $\delta$ and $-0.8 \%$ for $\beta)$. This strain is well within other observations of these phases and consistent with thermal expansion mismatch over the ca. $800{ }^{\circ} \mathrm{C}$ quench from high-temperature formation to subsequent room-temperature measurements; the database entries for $\delta$ reflect patterns collected at high temperature since the phase was not previously accessible at room temperature. The fraction of polymorphs present in each diffraction pattern was determined by multi-peak fitting and scaled relative to phase-pure patterns. The peaks fit are listed in Table S1. These peaks were simultaneously fit using Gaussian peak functions (in the case of the $\alpha$-phase 111 and -112 peaks, the location is so similar a single Gaussian was used). This fitting was performed using custom GENPLOT (Computer Graphics 
Service, Ithaca, NY) code. Phase intensities plotted in manuscript are the intensities of the peaks belonging to $\alpha$-phase $120, \beta$-phase 002 , and $\delta$-phase 002 . These peaks were chosen because the highest peak of all three phases overlap substantially, making differentiation of phases difficult. This selection over-estimates the $\alpha$-phase due to the use of the more intense 120 peak, but no other $\alpha$-peak had sufficient signal above background to be used, and this overestimation of $\alpha$ does not undermine the thrust of this work focused on metastable $\delta$ and $\beta$-phases. This work does not constitute a Rietveld refinement of the data, which was made difficult by the small nucleate sizes and the backgrounds both instrumental and due to the $\mathrm{SiO}_{2}$ and $\mathrm{Si}$ underlayers.

Table S1: Peaks fit as part of multi-peak fitting and phase deconvolution.

\begin{tabular}{|c|c|c|c|}
\hline Amorphous & $\alpha$-Phase & $\beta$-Phase & $\delta$-Phase \\
\hline Amorphous peak & 120 & 111 & 111 \\
\hline & 111 and -112 & 220 & 002 \\
\hline & & 002 & \\
\hline
\end{tabular}

For Figure 3a, if a single phase's signal was more than $25 \%$ higher than all other phases, it was designated as dominant. If two phases had intensities within $25 \%$, then the sample was identified as mixed phase. Fits in Figure 2d were developed using even polynomials to force symmetry of the fit for equivalent annealing conditions on either side of the laser scan, and were transformed into temperature for Figure 2e using lgLSA calibrations. 3D surfaces used to create contour plots in Figure 3b-d were developed using fitting shown in Figure 2e. The reported limit on coherent scattering domain size was determined using the Debye-Scherrer equation assuming zero instrumental broadening and, as such, represents a lower limit on domain size. Prolonged anneals above the equilibrium melt resulted in damage in the form of an amorphous structure with a shifted 
amorphous peak compared to the as-deposited amorphous film, likely due to the well reported instability of $\mathrm{Bi}_{2} \mathrm{O}_{3}$ in low oxygen partial pressure environments at high temperatures (20).

\section{Scanning Electron Microscopy}

The morphology of the annealed areas was examined using a Tescan Mira3 scanning electron microscope (SEM) using an in-lens detector and an accelerating voltage of $15 \mathrm{kV}$. The laser scan image in Figure $1 \mathrm{~d}$ had a $1 \mathrm{~ms}$ dwell and $900{ }^{\circ} \mathrm{C}$ peak temperature.

X-Ray Photoelectron Spectroscopy (XPS)

XPS measurements were performed on a Surface Science Instruments SSX-100. The resulting data were charge-compensated by adjusting the position of the adventitious $\mathrm{C} 1 \mathrm{~s}$ peak to $284.8 \mathrm{eV}$. The composition results were determined by integrating the $\mathrm{O} 1 \mathrm{~s}$ peak (deconvolved from adsorbed carbonate contributions) and the $\mathrm{Bi} 4 \mathrm{f} 5 / 2$ peak; the $\mathrm{Bi}$ 4f $7 / 2$ peak was not used due to possible contributions from the Bi 5 s shell.

\section{Large Area Anneals}

Large area anneals were created by using multiple offset line anneals where line anneals were offset $50 \mu \mathrm{m}$ center-to-center over $40 \mathrm{~mm}$ wide areas (801 independent anneals). Each anneal stripe used the lgLSA conditions of $900{ }^{\circ} \mathrm{C}$ peak temperature and $250 \mu$ s dwells. As the annealing front moves across the annealed area, previously annealed areas experience $250 \mu \mathrm{s}$ dwells at lower and lower temperatures until the annealing front has moved too far away to impact the material $\approx \approx$ $2 \mathrm{~mm}$ ). A diffraction pattern from this area annealed $\delta$-region is shown in Figure S3 along with expected peak positions for the computationally predicted oxygen-ordered $\delta$-phase $(24,25)$. The diffraction pattern in Figure S3 was taken with a copper X-ray lab source (Bruker General AreaDetector Diffraction System equipped with a Bruker Hi-Star area detector). Patterns were radially integrated using the supplied instrument software. The primary X-ray energy was copper $\mathrm{K}_{\alpha}$, but 
near the strong peak at $28^{\circ}$ a doubling occurs due to $\mathrm{K}_{\beta}$ at $25.2^{\circ}$. Measured peak intensities differed from predicted intensities, likely due to partial texturing of these thin films. We believe that these suspected oxygen order peaks are visible in the area anneals, but not in the single line anneals earlier reported, due to nucleation and Ostwald-like ripening of ordered oxygen domains arising during the extended area anneals. Specifically, we speculate that re-annealing of low-temperature $\delta$-phase for short dwells (as occurs during overlapped laser scans) is key to creating this large range oxygen ordered $\delta$-phase. While X-ray data confirmed the $\delta$-phase, morphological instabilities during the overlapped laser anneals resulted in grooves through the full thickness of the $\mathrm{Bi}_{2} \mathrm{O}_{3}$ which prevented electrical measurements.

\section{Raman Spectroscopy}

Raman spectra were measured using a WITec Alpha 300 Raman microscope equipped with a 100x objective lens, a 1800 lines/mm grating, and a $488 \mathrm{~nm}$ probe laser. The beam was focused to give a measurement spot size of approximately $300 \mathrm{~nm}$. Traces in Figure S2 are the sum of 21 consecutive measurements collected at 1 um spacing to better approximate the XRD probe volume; measurements did not exhibit significant differences from their sum other than lower signal-to-noise ratio. 


\section{Additional References}

28. J. Ilavsky, Nika: Software for two-dimensional data reduction. J. Appl. Crystallogr. 45, 324-328 (2012).

29. E. W. Bohannan, C. C. Jaynes, M. G. Shumsky, J. K. Barton, J. A. Switzer, Lowtemperature electrodeposition of the high-temperature cubic polymorph of bismuth(III) oxide. Solid State Ionics. 131, 97-107 (2000).

30. H. T. Fan, X. M. Teng, S. S. Pan, C. Ye, G. H. Lia, and L. D. Zhang, Optical properties of $\delta$-Bi2O3 thin films grown by reactive sputtering. Appl. Phys. Lett. 87, 231916 (2005).

31. O. Depablos-Rivera, A. Martínez, S.E. Rodil, Interpretation of the Raman spectra of bismuth oxide thin films presenting different crystallographic phases. J. Alloys and Compounds 853, 157245 (2021). 


\section{Supplementary Figures}

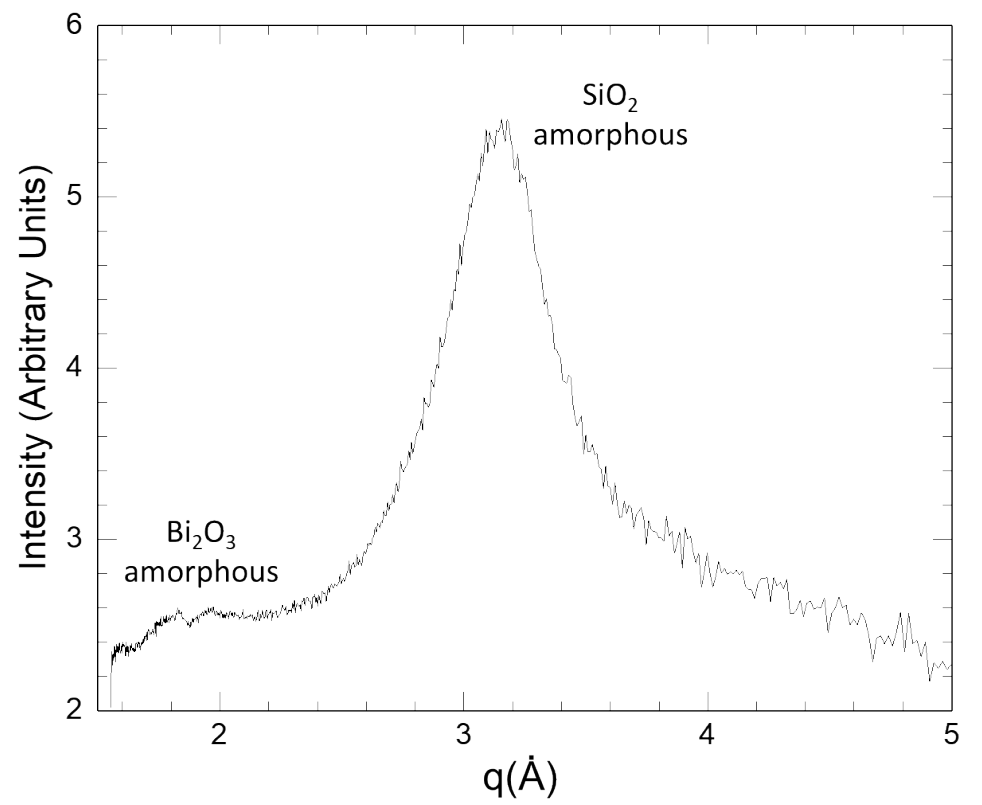

Figure S1. X-ray diffraction pattern of as-deposited $\mathrm{Bi}_{2} \mathrm{O}_{3}$ on $\mathrm{SiO}_{2}$ buffer layer on silicon single crystalline wafer. 


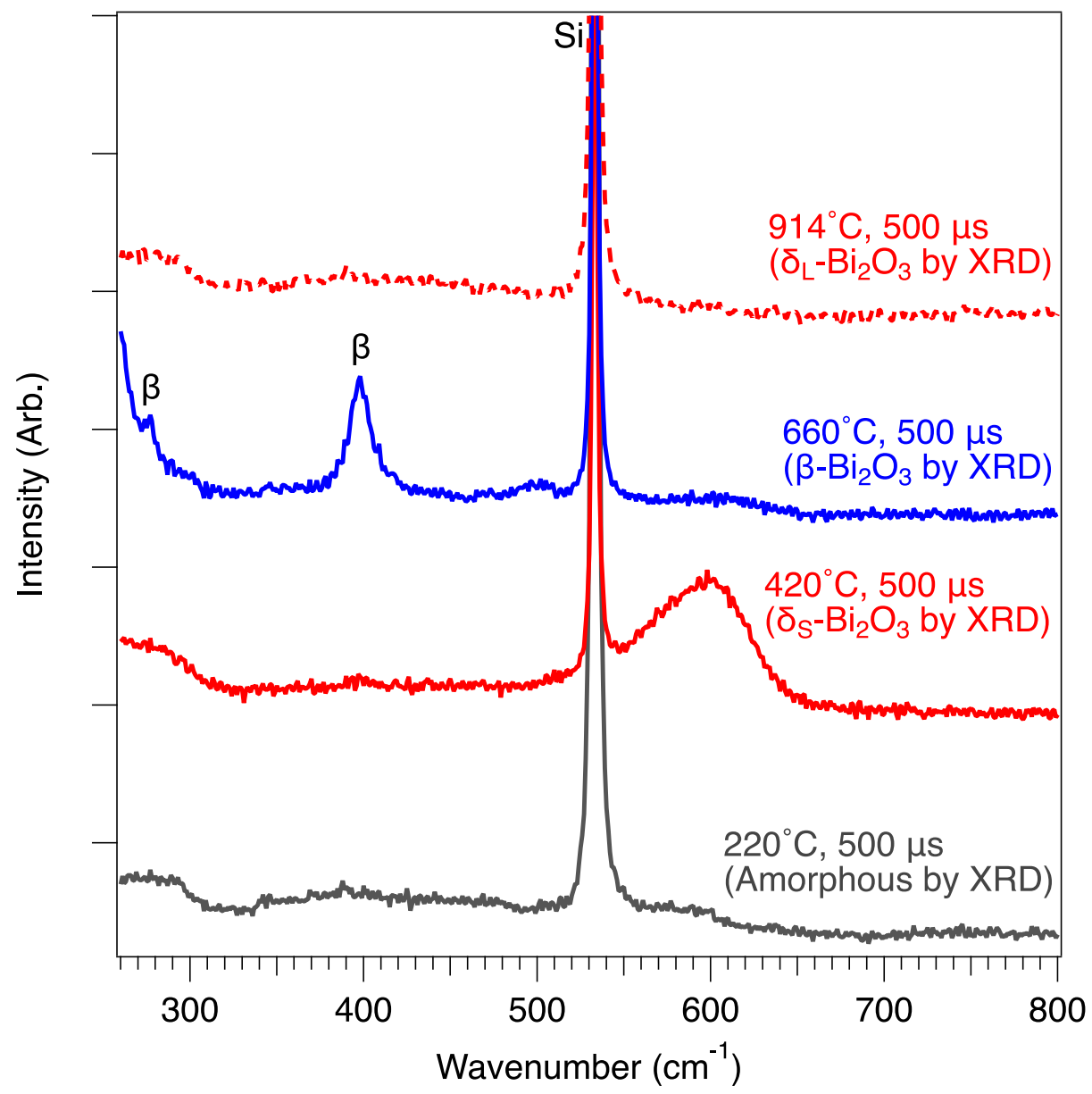

Figure S2. Representative Raman spectra of points in a $500 \mu$ s laser scan, demonstrating phase purity. Peak assignments indicated in the figure are from ref. 30 and 31 . Peak at $600 \mathrm{~cm}^{-1}$ has been attributed to $\delta$-phase previously but our work agrees with 31 in showing $\delta$-phase with larger grains annealed at higher temperatures appear to lose this $600 \mathrm{~cm}^{-1}$ peak. 

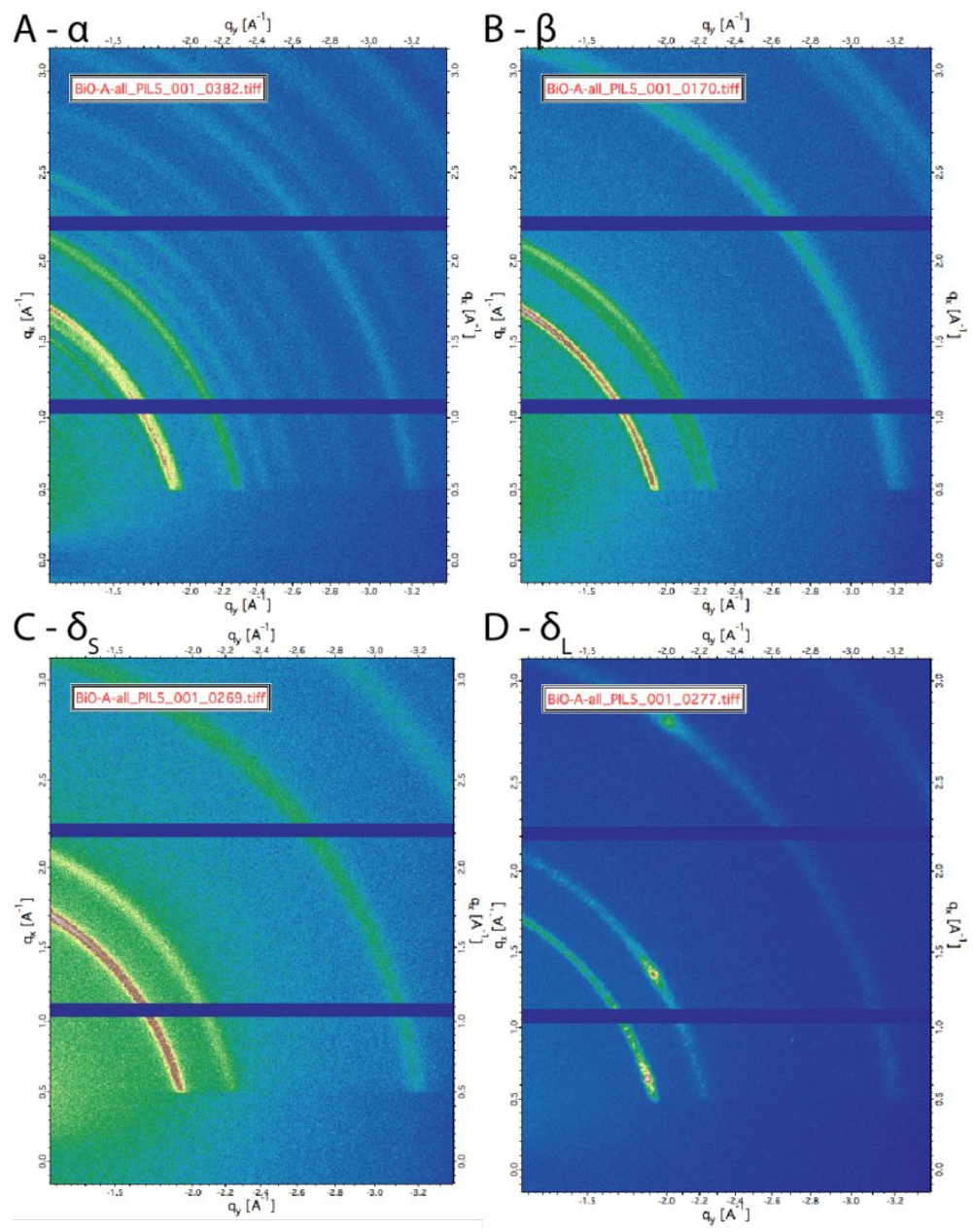

Figure S3. Two-dimensional X-ray diffraction patterns corresponding to data shown in Fig. $2 \mathrm{C}$, indicating a lack of macroscopic orientation in $\alpha, \beta$, and $\delta_{S}$, while $\delta_{L}$ exhibits spots consistent with textured crystallization. 

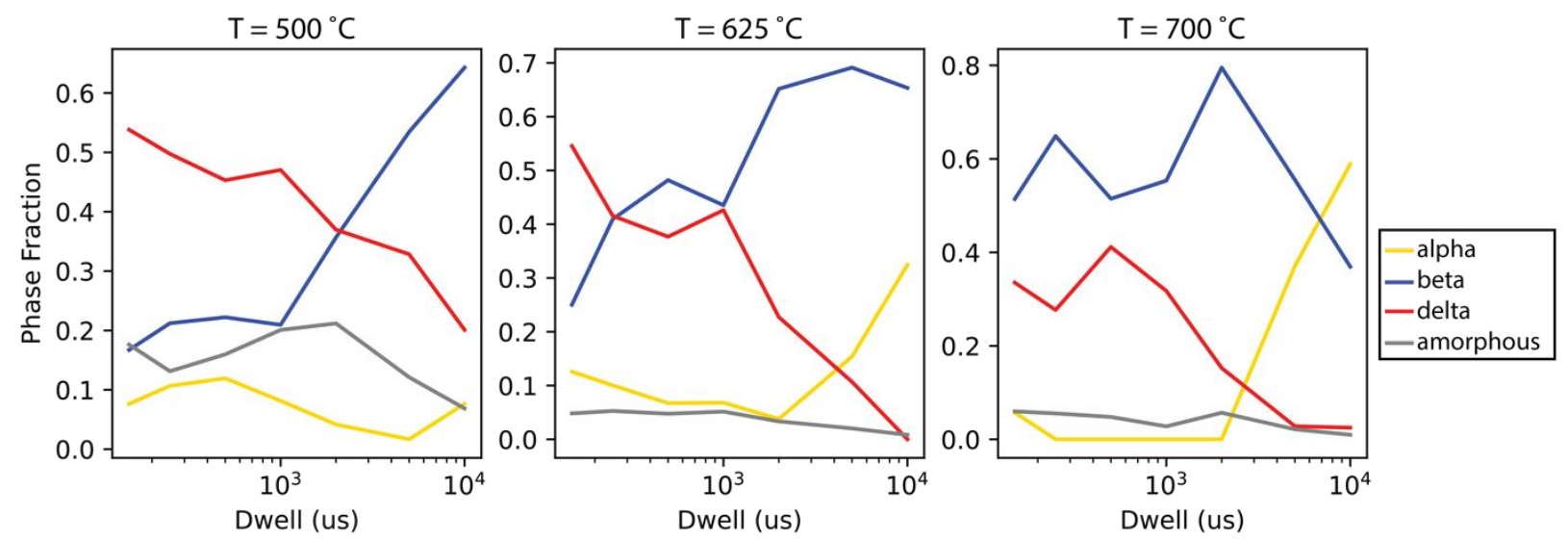

Figure S4. Phase fraction as a function of dwell time at $500{ }^{\circ} \mathrm{C}, 625^{\circ} \mathrm{C}$, and 700

${ }^{\circ} \mathrm{C}$. Delta (red) and beta (blue) interchange at short and intermediate dwell, with alpha developing from beta at the highest temperatures and longest dwells. 
A

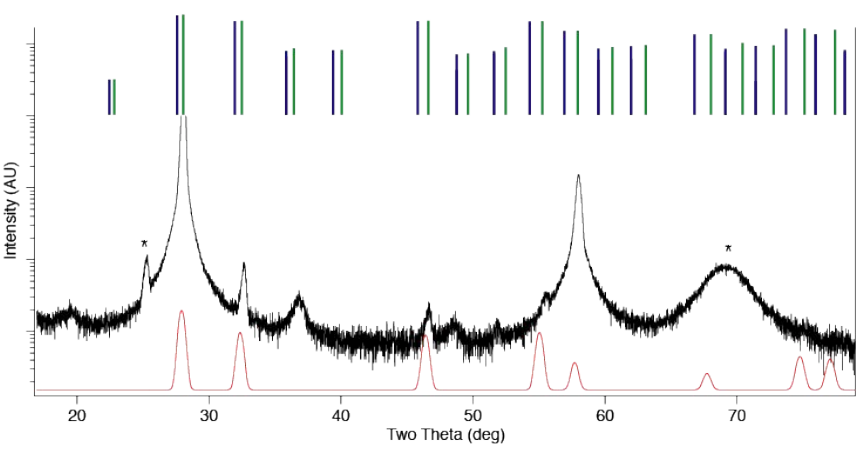

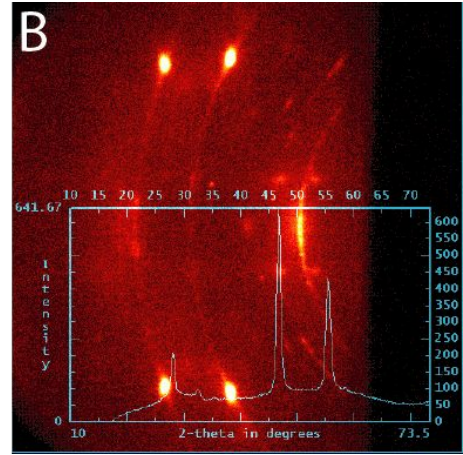

Figure S5. (A) Diffraction pattern of area-annealed $\delta$ - $\mathrm{Bi}_{2} \mathrm{O}_{3}$ sample (black) compared to simulated pure $\delta-\mathrm{Bi}_{2} \mathrm{O}_{3}$ in red (29) and computationally predicted oxygen-ordered $\delta$ - $\mathrm{Bi}_{2} \mathrm{O}_{3}(24)$ both in the zero-stress state simulated (blue) and strained to match the $\delta$ - $\mathrm{Bi}_{2} \mathrm{O}_{3}$ peaks that we observe (green). The area-annealed $\delta$ $\mathrm{Bi}_{2} \mathrm{O}_{3}$ exhibits peaks corresponding to all of the disordered $\delta-\mathrm{Bi}_{2} \mathrm{O}_{3}$ peaks, plus additional peaks from symmetry breaking particularly noticeable in the (024), (006), and (062) reflections at ca. $36^{\circ}, 49^{\circ}$, and $54^{\circ}$. The broad peak near $70^{\circ}(*)$ is due to the $\mathrm{SiO}_{2}$ underlayer and the peak near $25.2^{\circ}(*)$ is a doubling of the primary $\delta$ peak caused by the $\mathrm{K}_{\beta} \mathrm{X}$-ray source. (B) Two-dimensional image that was integrated to produce the experimental trace in (A). The relatively low number of observed peaks, and deviations from expected intensity, are likely due to a high degree of texture in the sample as indicated by the diffraction spots rather than clear rings. 

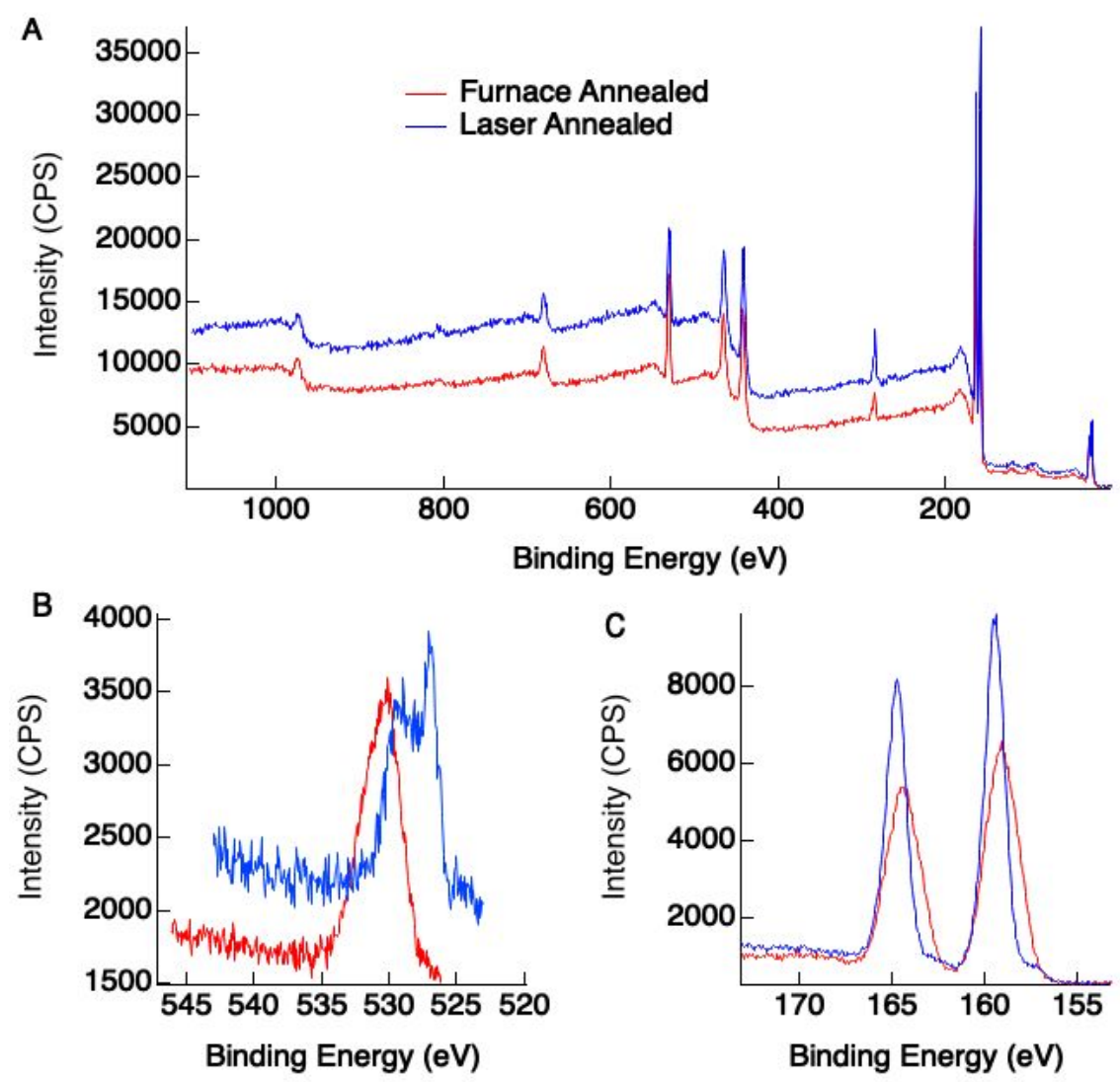

Figure S6. X-ray photoelectron spectroscopy of furnace annealed (red) and laser annealed (blue) $\mathrm{Bi}_{2} \mathrm{O}_{3}$. The survey scan (A) and high-resolution bismuth $4 \mathrm{f}(\mathrm{C})$ scan do not show substantial differences between the two samples, consistent with no change in the stoichiometry between the two conditions. The origin of a $1 \mathrm{eV}$ to $2 \mathrm{eV}$ shift in the high-resolution $\mathrm{O} 1 \mathrm{~s}$ (B) scan is unknown, but may be related to sample charging. The sharp peak at ca. $527 \mathrm{eV}$ in the laser-annealed oxygen is attributed to carbonate adsorption. 